\title{
1 Epigenetic Instability May Alter Cell State Transitions and Anticancer Drug
} Resistance

Abstract

8 Drug resistance is a significant obstacle to successful and durable anti-cancer

9 therapy. Targeted therapy is often effective during early phases of treatment;

10 however, eventually cancer cells adapt and transition to drug-resistant cells states

11 rendering the treatment ineffective. It is proposed that cell state can be a determinant

12 of drug efficacy and manipulated to affect the development of anticancer drug

13 resistance. In this work, we developed two stochastic cell state models - referenced

14 to brain tumors - that included transcriptionally-permissive and -restrictive states

15 based on the underlying hypothesis that epigenetic instability mitigates lock-in of

16 drug-resistant states. One model used single-step state transitions, whereas the other

17 considered a multi-step process to lock-in drug resistance. The latter model showed

18 that with moderate epigenetic instability the drug-resistant cell populations were

19 reduced, on average, by $60 \%$, whereas a high level of epigenetic disruption reduced

20 them by about $90 \%$. Generation of epigenetic instability via epigenetic modifier

21 therapy could be a viable strategy to mitigate anticancer drug resistance. 


\section{Introduction}

23 The hallmarks of cancer have evolved from their original inception and indicate the

24 complexity of the disease and the remarkable adaptability of cancer cells to sustain

25 growth under adverse conditions including drug therapy [1, 2]. Epigenetic

26 reprogramming or plasticity is part of the adaptability armamentarium of cancer cells

27 that alters gene expression by regulating gene transcription through histone post-

28 translational modifications (PTMs). Thus, disrupted epigenetic mechanisms play a

29 pivotal role in cancer biology that are characterized by epigenetic plasticity and

30 altered cell states [3, 4]. In recent years, epigenetic modifiers have been used in

31 attempts to reverse resistance to other drugs through epigenetic modifications [5-

32 10]. The reprogramming capability of the epigenome indicates that cells are

33 susceptible to disequilibrium and it is precisely this instability that may be tapped to

34 offer a therapeutic strategy to improve drug therapy.

35 We previously presented a deterministic cell state model of mutant isocitrate

36 dehydrogenase-1 (IDH1) gliomas that consisted of quiescent, stem and differentiated

37 glioma cells that could transition between each other reflecting intratumoral

38 heterogeneity (ITH) and cellular adaptation [11]. The model incorporated cell

39 proliferation, death, and state transitions in the context of drug resistance. Model

40 simulations indicated that modulation of cell transition rates based upon fluctuating

41 D-2-hydroxy-glutatarate (D2HG) concentrations, a known epigenetic modifier of 
42 this type of brain tumor, partially mitigated the evolution of drug-resistant tumors.

43 However, the intrinsic randomness of cell biology is more appropriately captured by

44 stochastic modeling approaches. Various studies have shown that models based on

45 Markovian stochastic transitions between tumor cell states are consistent with

46 observed attributes of tumor heterogeneity [12-14]. In this investigation, the

47 stochastic cell state models consider epigenetic-mediated transcriptionally-

48 permissive and transcriptionally-restrictive states as precursors to drug resistant

49 states. The mathematical models show that implementation of epigenetic instability

50 may be a strategy to subdue the evolution of drug resistance.

52 Results

53 Basic Single-Step Model

54 A solid tumor, and herein referred to as a brain tumor or glioblastoma multiforme

55 (GBM), was considered to consist of multiple cell types or states (Figure 1). The cell

56 populations change over time due to proliferation, death, and state transitions. State

57 transitions may be initiated due to the microenvironment (blood flow and hypoxia)

58 and drug therapy as a means to survive and grow. In the model (Figure 1), at time

59 zero, there are five cell states wherein both differentiated glioma $(\mathrm{G})$ and glioma

60 stem cells (GS) exist in two transcriptional states, either permissive (Tp) or

61 restrictive (Tr) that leads to four cell states (G-Tp, G-Tr, GS-Tp, and GS-Tr). 
62 Quiescent (Q) cells comprise a fifth cell state. Drug therapy can also lead to two

63 more cell states, drug resistant glioma (G-R) and glioma stem cells (GS-R). Cells in

64 a particular state can undergo either birth, death or transition to another cell state,

65 except Q cells that may only die or transition. This construction of the model permits

66 an understanding of how epigenetic changes affect cell state dynamics. The first set

67 of simulations are shown in Figure 2 using the parameters listed in Table 1 that

68 indicate birth, death and cell transition rates for each cell type under different

69 conditions.

A

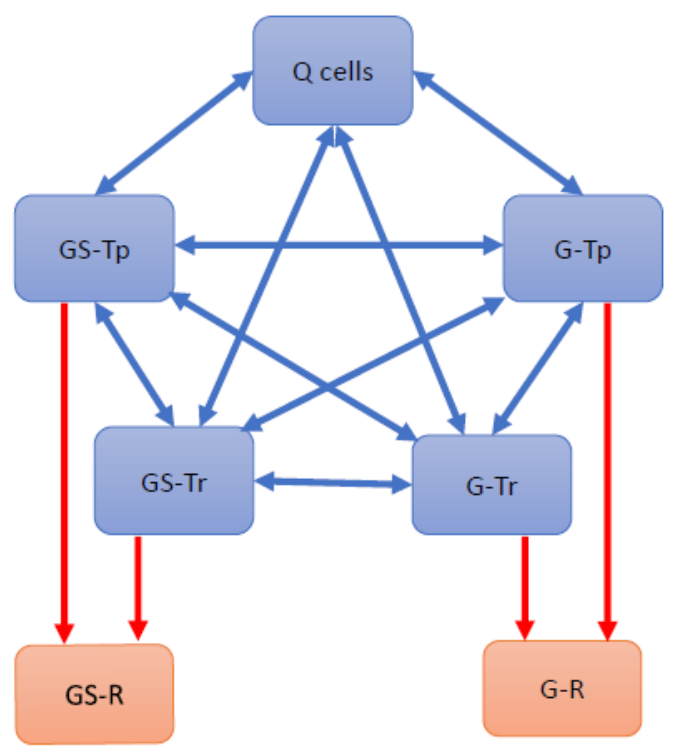

B

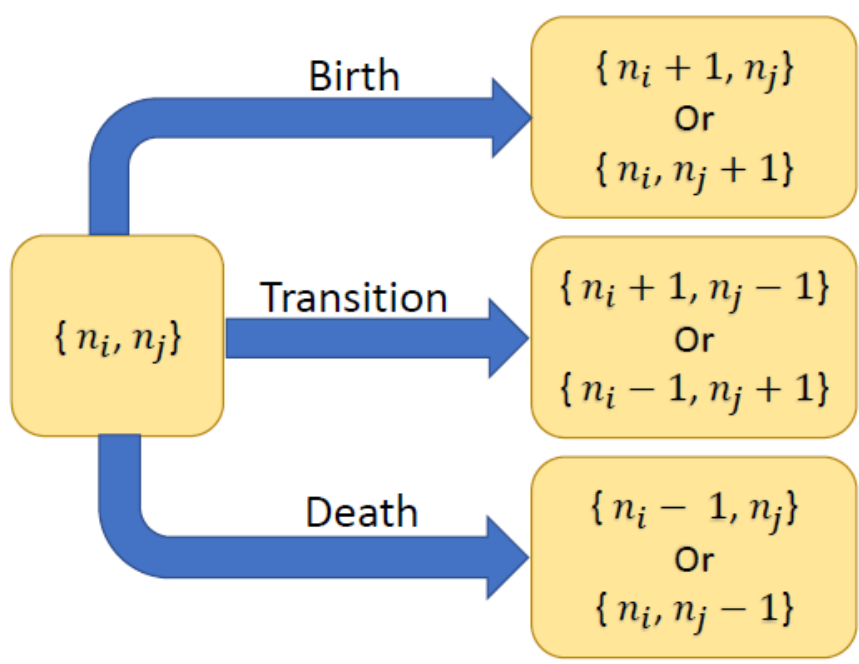

$i, j:$ Any two cell states

$\left\{n_{i}, n_{j}\right\}:$ Number of cells in $\mathrm{i}^{\text {th }}$ and $\mathrm{j}^{\text {th }}$ cell states. 
71 Figure 1: Schematic of the basic single-step cell state model. (A) The model

72 consists of five states: Quiescent cells (Q), transcriptionally-permissive Glioma and

73 Glioma Stem cells (G-Tp and GS-Tp respectively), and transcriptionally-restrictive

74 glioma and glioma stem cells (G-Tr and GS-Tr respectively). Cells can transition

75 between any two of these states (indicated by two-sided blue arrows). In addition, in

76 the presence of drug therapy, cells may transition to drug resistant states (G-R/GS-

$77 \mathrm{R})$. Once in a resistant cell state, the cells are unable to transition back to drug-

78 sensitive states (indicated by one-way red arrows). (B) Cells can undergo birth

79 (except Q cells), death or transition to another state.

80

\begin{tabular}{|c|c|c|c|c|c|c|c|c|c|c|c|}
\hline \multirow[t]{2}{*}{ Condition } & \multirow[t]{2}{*}{$\begin{array}{c}\text { Time } \\
(\mathbf{t})\end{array}$} & \multicolumn{3}{|c|}{ Q Cells } & \multicolumn{3}{|c|}{$\begin{array}{c}\text { G-Tp, G-Tr, GS-Tp, } \\
\text { GS-Tr }\end{array}$} & \multicolumn{3}{|c|}{ G-R, GS-R } & \multirow[t]{2}{*}{$\begin{array}{l}\text { Figure } \\
\text { Number }\end{array}$} \\
\hline & & BR & DR & TR & BR & DR & TR & BR & DR & TR & \\
\hline $\begin{array}{l}\text { Control } \\
\text { No drug }\end{array}$ & & 0 & $\mathrm{n}$ & $0.1 *_{n}$ & $2 * \mathrm{n}$ & $\mathrm{n}$ & $0.1 * n$ & NA & NA & NA & Fig. 2A \\
\hline \multirow[t]{2}{*}{ Drug sensitive } & $t<2$ & 0 & $\mathrm{n}$ & $0.1 * n$ & $2 * n$ & $\mathrm{n}$ & $0.1 * n$ & NA & NA & NA & \multirow[b]{2}{*}{ Fig. 2B } \\
\hline & $t>2$ & 0 & $2.2 * n$ & $0.1 * n$ & $2 * n$ & $2.2 * n$ & $0.1 * n$ & NA & NA & NA & \\
\hline \multirow{2}{*}{$\begin{array}{l}\text { Drug resistant } \\
\text { with stable } \\
\text { transitions }\end{array}$} & $t<2$ & 0 & $\mathrm{n}$ & $\mathrm{n}$ & $2 * n$ & $\mathrm{n}$ & $\mathrm{n}$ & NA & NA & NA & \multirow[b]{2}{*}{ Fig. 2C } \\
\hline & $t>2$ & 0 & $2.2 * n$ & $\mathrm{n}$ & $2 * n$ & $2.2 * \mathrm{n}$ & $\mathrm{n}$ & $2 * n$ & $\begin{array}{l}2.2 * n * \\
e^{\frac{-(t-2)}{t_{\max }}}\end{array}$ & $\mathrm{n}$ & \\
\hline \multirow{2}{*}{$\begin{array}{l}\text { Drug resistant } \\
\text { with unstable } \\
\text { transitions }\end{array}$} & $t<2$ & 0 & $\mathrm{n}$ & $\mathrm{n}^{*} \mathrm{u}$ & $2 * n$ & $\mathrm{n}$ & $\mathrm{n}^{*} \mathrm{u}$ & NA & NA & NA & \multirow[b]{2}{*}{ Fig. 2D } \\
\hline & $t>2$ & 0 & $2.2 * \mathrm{n}$ & $\mathrm{n}^{*} \mathrm{u}$ & $2 * n$ & $2.2 * n$ & $\mathrm{n}^{*} \mathrm{u}$ & $2 * n$ & $\begin{array}{l}2.2 * n \\
* e^{\frac{-(t-2)}{t_{\max }}}\end{array}$ & $\mathrm{n}^{*} \mathrm{u}$ & \\
\hline
\end{tabular}

81 Table 1: Summary of the model simulation parameters. The various conditions

82 and parameter values for each cell type, where $\mathrm{n}$ indicates the number of cells in a 
83 state; $\mathrm{u}$ is a random number parameter generating instability in the transitions; $\mathrm{t}$ is

84 the time elapsed in the system; and $t_{\max }$ is the maximum time allowed in the

85 simulations. BR: birth rate, DR: death rate and TR: transition rate.

87 Under control conditions (without drug) and minimal cell state transitions (Figure

88 2A), cells grow exponentially without large changes in the population of Q cells.

89 This leads to a dynamically-balanced population where none of the cell states are

90 dominant. In Figure 2B, drug-sensitive cell population dynamics are shown upon the

91 addition of a drug with the condition that drug resistant populations are not allowed.

92 Initially, there is exponential growth similar to the control conditions, followed by a

93 decrease in all cell populations after the drug is introduced at 2 days since there are

94 no transitions into resistant states. In control and drug sensitive cases (Figures 2A

95 and $2 \mathrm{~B})$, the transition rates are minimal at all times $\left(0.1^{*} \mathrm{n}\right)$. When transitions to

96 drug resistant states are allowed the cell dynamics change accordingly (Figure 2C).

97 Prior to addition of the drug, cells in all states grow exponentially like in Figure 2A

98 and 2B, without any dominant cell state. Now, as drug resistance evolves, all

99 sensitive cell state populations decrease at the expense of an increase in resistant cell

100 state populations. This happens because sensitive cells can transition into resistant

101 cells but the reverse cannot happen. In addition, the death rate of resistant cells

102 decreases with time (due to acquiring resistance) but the birth rate remains the same. 
103 Hence, unless the entire population of resistant cells die, they grow exponentially

104 later. Due to the important role of birth and death rates of resistant cells (apart from

105 transition rates among all cell states) in the model dynamics, we investigated their

106 effect on population growth (Supplement section 1, Table S1). As expected, a higher

107 birth rate of resistant cells leads to an increase in their population. Similarly, a higher

108 death rate leads to a decrease in the resistant cell population. A high rate of transition

109 keeps the cell population evenly distributed among the cell states (Supplementary

110 Figure S1).

111 When epigenetic instability is introduced in the transition rates via a stochastic

112 parameter (see Table 1) simultaneously with drug exposure there is a reduction in

113 the overall population of resistant cells due to the random changes in the transition

114 rates (Figure 2D). Since the simulations shown in Figure 2A-D represent single

115 population trajectories, we performed a large number of simulations (2000) in order

116 to understand the overall behavior of the model (Figure 2E). The average resistant

117 cell population with or without epigenetic instability agree with the results from

118 Figures 2A-D, and indicate that epigenetic instability reduces the resistant cell

119 population size by $35 \%$. 

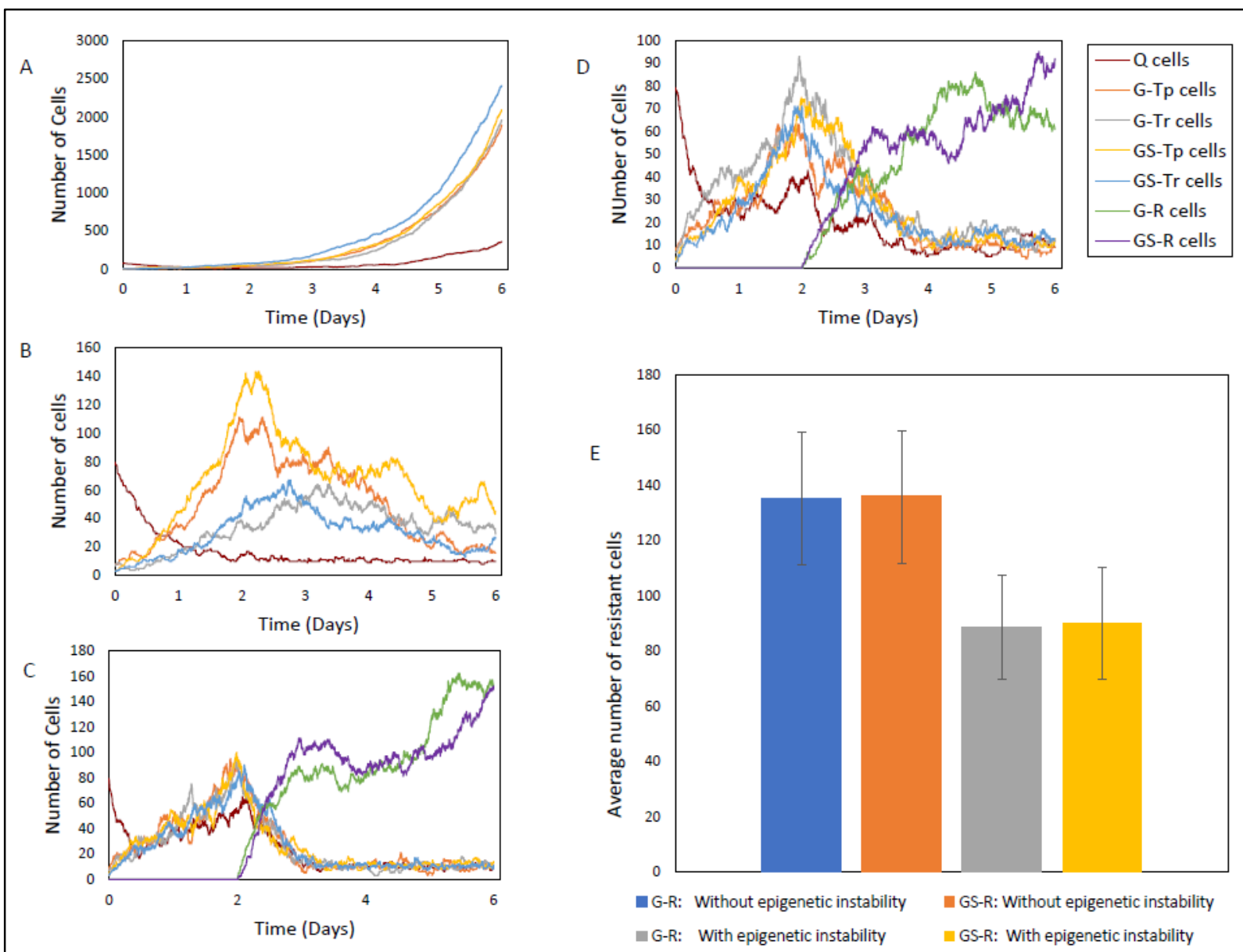

124 Figure 2: Cell population dynamics for the basic single-step model. (A) Control

125 with low transition rates and no drug exposure. (B) Drug-sensitive cells with drug

126 introduced at $\mathrm{t}=2$ days in the absence of drug resistance. (C) Drug-resistant cells,

127 with sensitive to resistant state transitions enabled upon addition of drug at $\mathrm{t}=2$

128 days. (D) Conditions same as $\mathrm{C}$ with epigenetic instability allowed upon drug

129 exposure at $\mathrm{t}=2$ days. (E) Average ( \pm standard deviations) resistant cell numbers at

$130 \mathrm{t}=6$ days based on 2000 independent simulations. Table 1 lists parameters for each

131 condition. 


\section{Epigenetic instability in the multi-step model}

134 The single-step basic model may not capture the complex transcriptional machinery

135 due to PTMs and associated changes in chromatin structure, and the requirement of

136 coordinated protein complexes to initiate gene transcription. Thus, a multi-step

137 model was developed that considered the transition from drug-sensitive cells to

138 drug-resistant as a multi-step lock-in process (Figures 3A and 3B). The lock-in

139 process was implemented as a 3-step switch wherein all three events must occur to

140 complete the transition from a sensitive to a resistant state (all switches must be On).

141 The probability of triggering the switch is equal for all switches $\left(p_{1}=p_{2}=p_{3}\right.$,

142 Figure 3B), and further, the triggering probability of each individual switch is

143 independent of the other switches. To apply epigenetic instability, the unidirectional

144 lock-in multi-step process is replaced by a bidirectional multi-step process (Figure

145 3B), where any of the three switches in an On state, can be turned Off.

146 The control and drug-sensitive conditions for the multi-step model are analogous to

147 the basic model, and thus, only the drug-resistant condition with and without

148 epigenetic fluctuations were considered. The forward multi-step lock-in process

149 leads to drug resistant states from both glioma stem cells and glioma cells (Figure

$1504 \mathrm{~A}$, Top panel). The parameters used to generate the simulations in Figure 4 are the

151 same as those used in Figure 2C; however, in the multi-step model the number of

152 resistant cells is reduced given the more complex lock-in process involved in 
153 completing a state transition. When epigenetic instability is introduced via the bi-

154 directional process (50\% probability of switch turning Off $\rightarrow$ On and vice versa), the

155 growth of resistant cells is hampered (40\% reduction) (Figure 4A, Middle panel).

156 Moreover, high epigenetic instability (80\% probability of switch turning On to Off),

157 led to a much larger reduction (90\%) in resistant cell populations (Figure 4A, Bottom

158 Panel). As with the basic model, conducing a large number of simulations (Figure

159 4B) indicates that moderate epigenetic instability reduces the resistant cell

160 population by about $60 \%$ (see Figure 4B). At high epigenetic disruption, the resistant

161 cell population is reduced by more than $90 \%$ compared to the scenario without any

162 epigenetic disruption.

163 In order to determine the effect of changing levels of epigenetic instability on the

164 resistant cell population, we performed simulations changing the probability of the

165 switches turning On $\rightarrow$ Off. As shown in Figure 4C, there is an inverse relationship

166 between the probability of a unit switch turning Off (a measure of epigenetic

167 instability) and the size of the resistant cell population. At the highest tested

168 probability of epigenetic disruption of $80 \%$, the resistance cell numbers declined by

169 more than $90 \%$. 
A

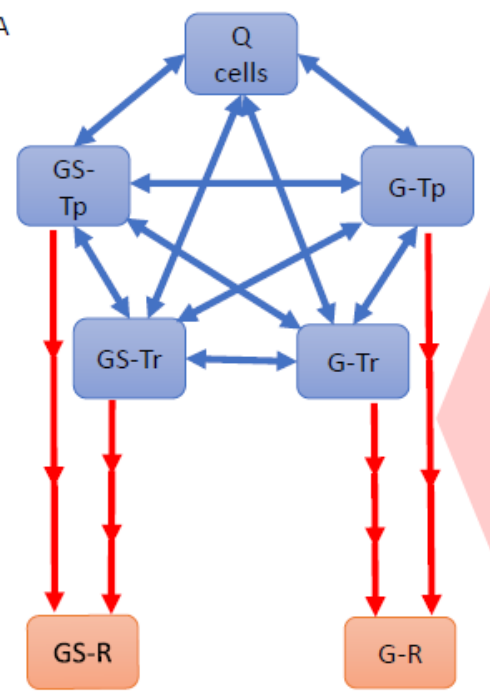

$\mathrm{B}$

\section{Multi-step epigenetic process}

Forward lock-in process Bi-directional process

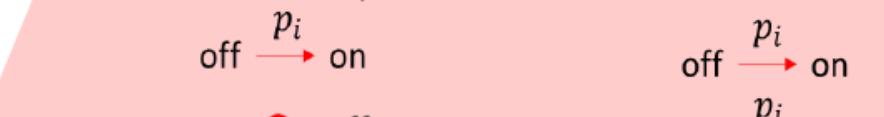

Sensitive

on $-\rightarrow$ off

on $\stackrel{p_{i}}{\longrightarrow}$ off
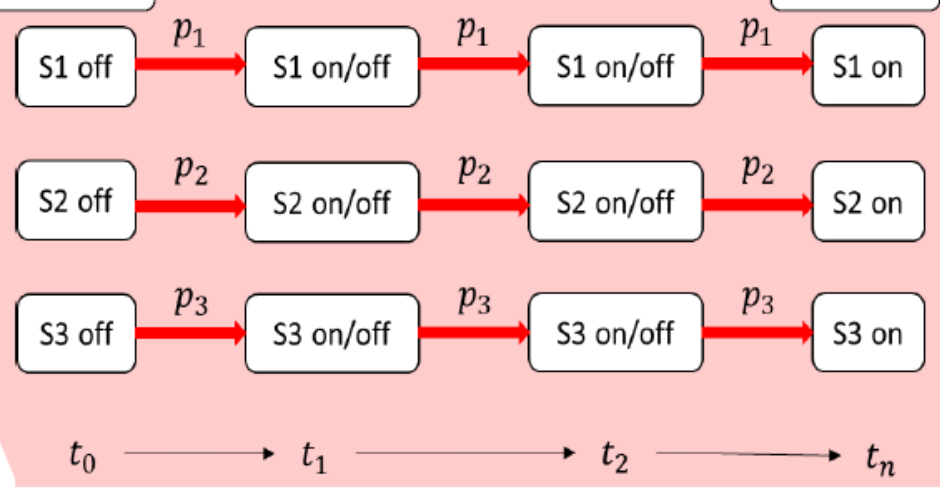

171 Figure 3: Schematic of the multi-Step model. (A) The multi-step model is similar

172 to the basic model (see Figure 1), but transitions to drug-resistant states are multi-

173 step processes (red arrows). Single-step processes are shown as blue-arrows. (B) The

174 three-step transition model is shown between sensitive and resistant cell states. The

175 forward lock-in process requires all three switches (S1, S2 and S3) to be On. The

176 bidirectional process allows any of the three switches to go from On $\rightarrow$ Off, thus

177 interrupting a transition to a resistant state. $\mathrm{p}_{\mathrm{i}}$ indicates the probability of the event

178 occurrence (switch turning Off $\rightarrow$ On or On $\rightarrow$ Off). 

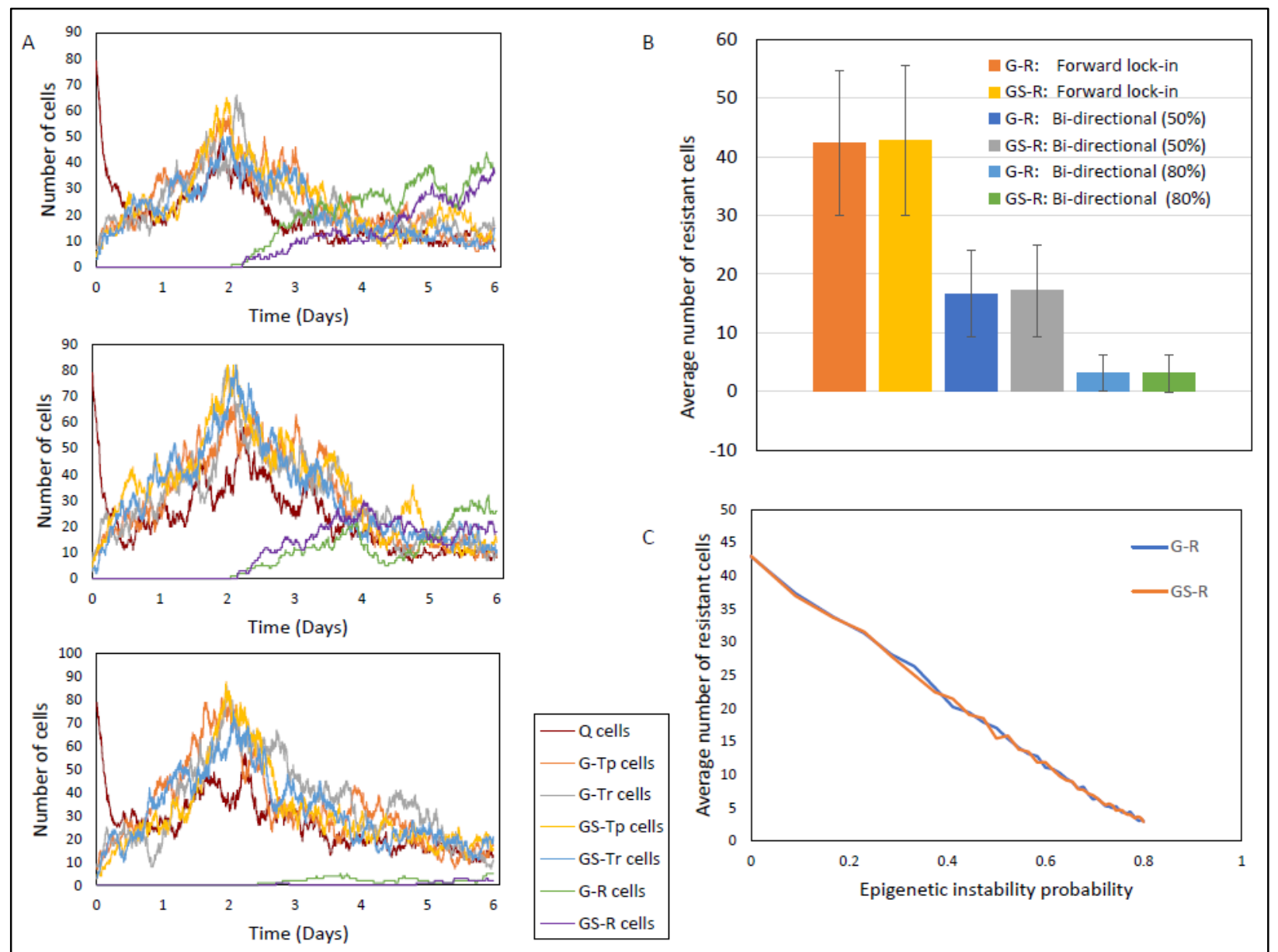

180 Figure 4: Cell population dynamics for the multi-step model. (A) Top panel:

181 Forward lock-in multi-step process with drug introduced at $\mathrm{t}=2$ days. Middle panel:

182 Bi-directional multi-step process with moderate epigenetic instability $(50 \%$

183 probability of switch turning On $\rightarrow$ Off). Bottom panel: Bi-directional multi-step

184 process with high epigenetic instability ( $80 \%$ probability of switch turning On $\rightarrow$ Off)

185 (B) Average ( \pm standard deviations) number of resistant cells at $\mathrm{t}=6$ days following 2000 independent simulations. The values $50 \%$ and $80 \%$ in the legend are the 187 probability of switch turning On $\rightarrow$ Off. (C) The average number of resistant cells 
188 (GS-R plus G-R) as a function of increasing epigenetic instability (probability of

189 switch turning On $\rightarrow$ Off) in a multi-step bi-directional process.

191 Discussion

192 Anticancer drug resistance has been studied for decades with the main solution being

193 the addition of drugs to circumvent resistance and restore cancer cell drug sensitivity

194 [15-20]. Genetic analyses of gene mutations and expression profiles are often the

195 basis of the drug resistance strategies that seek to identify the drug resistant-inducing

196 genes and proteins, which may provide viable drug targets. Although the approach

197 has appeal given the mechanistic justification, and has produced a plethora of drug

198 combinations, these approaches ultimately fail since they do not consider

199 intratumoral heterogeneity and innate differences in cell sensitivity to drugs, as well

200 as cellular adaptation including the role of epigenetic reprogramming [21].

201 Both deterministic and stochastic mathematical models have been employed to study

202 intratumoral heterogeneity and the emergence of drug resistance in cancer [22-27].

203 Among deterministic approaches, the most common use ODEs to model population

204 growth of tumors [28]. Stochastic approaches, although computationally taxing, are

205 generally more appropriate for modeling biological systems, which are naturally

206 noisy and replicate the dynamics of gene transcription. In particular, discrete

207 stochastic processes are quite relevant in modeling tumor growth as they can predict 
208 the probability of development of at least one resistant cell in a tumor [29-31].

209 Stochastic differential equations are used to model tumor heterogeneity as they

210 provide a robust methodology for mechanistic modeling and the control of noise [32-

$21133]$. The stochastic modeling approach we employed is common to that used in

212 evolutionary dynamics based on branching processes with Markovian properties that

213 are simulated with the Gillespie algorithm [34, 35].

214 In this work, stochastic cell state models were developed to understand the effect of

215 epigenetic fluctuations on tumor heterogeneity and drug resistance. Both the single-

216 step and multi-step models show exponential growth of resistant cells upon drug

217 exposure (Figures 2C and 4A, Top Panel). However, when epigenetic instability was

218 introduced in either model, the resistant cell populations decreased, and were

219 inversely proportional to the probability of the multi-step switches turning Off

220 (Figure 4C). In the single-step model, the reduction due to epigenetic instability is

221 about 35\%. In the multi-step model, at moderate epigenetic instability (50\%

222 probability of a switch turning Off), the reduction in resistant cells was about $60 \%$

223 whereas at high epigenetic instability ( $80 \%$ probability of a switch turning Off) the

224 reduction is more than $90 \%$.

225 Both the single-step and multi-step models specify two likely epigenetic states,

226 either transcriptionally permissive or restrictive. In the current set of simulations,

227 there is no assigned preference to either state in terms of contributing to or mitigating 
228 drug resistant states. As such, either transcriptional state has an equal probability to

229 transition to a resistant state. Although knowledge of specific histone PTMs that are

230 sought for disruption may be advantageous in designing epigenetic modifier therapy,

231 it is posed that such drug therapy cycles between transcriptionally-permissive and

232 transcriptionally-restrictive states to maintain instability. Constant drug dosing rates

233 are averse to instability, and lend themselves to stability in terms of the cellular

234 programs that trigger drug resistance. Thus, although epigenetic modifiers are a

235 route to alter histones PTM, their dosing schedules should be variable to support

236 instability and minimize cell adaptation.

237 Our approach of combating anticancer drug resistance through generation of

238 epigenetic instability may have advantages over prevailing methods. First, it reduces

239 the complexity of ITH at least in terms of epigenetic plasticity as consisting of either

240 transcriptionally-permissive or -restrictive cell states. This simplification - currently

241 treated as an agnostic feature - may be tuned to repress oncogenes or to activate

242 tumor suppressor genes by integrating the cell state model with mechanistic

243 biochemical models. Albeit, the tuning of drug therapy to repress or activate desired

244 genes through epigenetic modifier therapy should retain a randomness to satisfy the

245 goal of instability. Second, a chaotic or constantly reprogramed epigenome may

246 limit cellular adaptations including those induced by constant drug exposures.

247 Current approaches to aggressively target one or more resistance pathways may 
248 select for alternate drug-resistant pathways to emerge. Although the resistant cells

249 are not extinguished at 50\% probability of the On/Off switch in the multi-step model,

250 they are not proliferating or are doing so very slowly. This attribute of epigenetic

251 instability - maintenance of this quasi-equilibrium between sensitive and drug-

252 resistant cells - could stall or prevent implementation of alternate drug-resistant

253 programs possibly rendering the cells susceptible to other treatments.

254 The proposed cell state models are theoretical and remain to be experimentally

255 tested. Prior to that, it may be beneficial to devise a hybrid stochastic-mechanistic

256 model to inform the most relevant experiments. For example, the task to implement

257 epigenetic instability therapy to mitigate temozolomide resistance in brain tumor

258 patients may be enhanced by considering synthesis of the DNA repair enzyme -

259 methylguanine methyltransferase (MGMT) that repairs the most lethal

260 temozolomide-induced O-6-methylguanine DNA adduct [36-38]. This would

261 involve identifying histone PTM(s) that facilitate transcription of MGMT (a

262 transcriptionally-permissive state) and then selecting epigenetic modifiers to prevent

263 this process, but to do so with a sporadic dosing regimen to abate continued MGMT

264 production whilst avoiding activation of alternate resistance mechanisms.

265 In conclusion, stochastic epigenetic instability models were developed that showed

266 the potential to mitigate the emergence of drug resistant cells. The models serve as

267 a foundation to explore detailed approaches to implement epigenetic instability 
268 therapy. It seems possible that either global or targeted epigenetic instability could

269 be sought with combinations of epigenetic modifiers and non-constant dosing

270 regimens. These aspirational goals will best be approached by computational models

271 and the current cell state model is a first-step in that direction.

273 Methods

\section{General Description of Cell State Model}

275 The mathematical details of the cell state models and how the simulations were

276 conducted are provided in the Supplement.

277 In the basic model, only single-step state transitions are allowed (see Figure 1). All

278 state transitions are reversible except those that culminate into a drug resistant state.

279 Once a cell is deemed drug resistant, it cannot transition to any other state. Integer

280 cell numbers are used for each cell state to discretize cell numbers within the tumor.

281 The system dynamics can be written in terms of a master ordinary differential

282 equation that governs the time evolution of the probability of the system occupying

283 a given cell state (Supplement section 2).

284 We incorporate stochasticity in our model by using a Monte Carlo methodology to 285 simulate birth, death, and state transitions. As quiescent cells do not proliferate, the

286 birth rate of Q cells is set to be zero. The death rates of quiescent cells were chosen

287 to be a proportional to the number of cells in the state and to incorporate the effect 
288 of drug, the death rate coefficients increases after the addition of drug (See Table 1).

289 Additionally, the Q cell population can increase or decrease due to state transitions

290 and the transition rates were set to be proportional to the number of cells in the state

291 from which the cell is transitioning.

292 For G and GS cell states, birth/death and transition rates are proportional to the

293 number of cells in that state. Before the addition of drug, the birth rate coefficient is

294 set higher than the death rate coefficient, which leads to exponential growth. After

295 the addition of drugs, the death rate is set higher than the birth rate to account for the

296 effects of drug, which leads to the decline in sensitive cells (Q cells, G and GS)

297 populations. Moreover, the birth rate and initial death rate of the drug resistant states

298 are kept the same as drug sensitive states. However, due to acquired drug resistance,

299 the death rate of resistant cells decays exponentially with time.

300 We considered cell transitions to resistant states under two different models. In the

301 basic model (see Figure 1), single-step one-way transitions occur from a drug

302 sensitive state to a resistant state. In the multi-step model (see Figure 3), three uni-

303 directional steps are required to "lock-in" a transition from a sensitive to a resistant

304 cell state. In both the basic and multi-step models, two cases - with and without

305 epigenetic instability - are considered.

306 For the simulations, we keep the initial total number of cells $=100$, among which

$307 \quad \mathrm{Q}=80 \quad$ GS-Tp $=3 \quad$ GS-Tr=3 $\quad$ GS-R $=0 \quad$ G-Tp=7 $\quad$ G-Tr $=7 \quad$ G-R $=0$. 
308 The drug is introduced in the system at time $\mathrm{t}=2$ days. In the single step model,

309 epigenetic instability or fluctuations in the transition rate is modeled using a random

310 number. All relevant parameters regarding each simulation is given in Table 1. To

311 avoid complete elimination of the sensitive cell population, we apply constraints on

312 the death rate and transition rates. If any sensitive cell state population is less than

31310 , then the death rate and transition rate to resistant cells is reduced to zero. All rate

314 coefficients have units Day $^{-1}$. A maximum time $\left(t_{\max }\right)$ of 20 days is allowed during

315 simulations. Additional details regarding the implementation of Gillespie algorithm

316 are provided in the supplement (section 3).

318 Acknowledgments

319 Funding from the State University of New York Empire Innovation program is

320 acknowledged for support of Anshul Saini, Ph.D.

\section{Competing interests}

323 There are no financial and non-financial competing interests related to this 324 investigation. 


\section{References}

329 1. Hanahan, D., \& Weinberg, R. A. (2000). The hallmarks of cancer. Cell, 100(1), $330 \quad 57-70$.

331 https://doi.org/10.1016/s0092-8674(00)81683-9 generation. Cell, 144(5), 646-674. https://doi.org/10.1016/j.cell.2011.02.013 and the hallmarks of cancer. Science (New York, N.Y.), 357(6348), eaal 2380. https://doi.org/10.1126/science.aal2380 Miller, T. E., Venteicher, A. S., Hebert, C. H., Carey, C. D., Rodig, S. J., Shareef,

343 S. J., Najm, F. J., van Galen, P., Wakimoto, H., Cahill, D. P., Rich, J. N., Aster, J.

344 C., Suvà, M. L., Patel, A. P., \& Bernstein, B. E. (2017). Adaptive Chromatin 345 Remodeling Drives Glioblastoma Stem Cell Plasticity and Drug Tolerance. Cell 346 stem cell, 20(2), 233-246.e7.

347 https://doi.org/10.1016/j.stem.2016.11.003 regulation in human cancer: the potential role of epi-drug in cancer therapy. Molecular cancer, 19(1), 79. https://doi.org/10.1186/s12943-020-01197-3 the Mechanisms by Which Epigenetic Modifiers Avert Therapy Resistance in

356 Cancer. Frontiers in oncology, 10, 992. https://doi.org/10.3389/fonc.2020.00992 
8. Zhao, Z., \& Shilatifard, A. (2019). Epigenetic modifications of histones in cancer. Genome biology, 20(1), 245. https://doi.org/10.1186/s13059-019-1870-5

9. Chiappinelli, K. B., Zahnow, C. A., Ahuja, N., \& Baylin, S. B. (2016). Combining Epigenetic and Immunotherapy to Combat Cancer. Cancer research, 76(7), 1683-1689. https://doi.org/10.1158/0008-5472.CAN-15-2125 10. Dunn, J., \& Rao, S. (2017). Epigenetics and immunotherapy: The current state of play. Molecular immunology, 87, 227-239. https://doi.org/10.1016/j.molimm.2017.04.012 pharmacometrics \& systems pharmacology, 7(9), 539-542. R Package to Quantify Stochastic Cell State Transitions. Bioinformatics and biology insights, 11, 1177932217712241.

392 Graeber, T. G., Ribas, A., \& Heath, J. R. (2017). Single-cell analysis resolves the

393 cell state transition and signaling dynamics associated with melanoma drug-

394 induced resistance. Proceedings of the National Academy of Sciences of the United

395 States of America, 114(52), 13679-13684.

396 https://doi.org/10.1073/pnas.1712064115 
15. Sun, X., \& Hu, B. (2018). Mathematical modeling and computational

401 https://doi.org/10.1093/bib/bbx065 S., Yaqubie, A., Kelly, N., Le, D. T., Lipson, E. J., Chapman, P. B., Diaz, L. A., Jr, Vogelstein, B., \& Nowak, M. A. (2013). Evolutionary dynamics of cancer in response to targeted combination therapy. eLife, 2, e00747. https://doi.org/10.7554/eLife.00747

17. Chapman, P. B., Hauschild, A., Robert, C., Haanen, J. B., Ascierto, P., Larkin, J., Dummer, R., Garbe, C., Testori, A., Maio, M., Hogg, D., Lorigan, P., Lebbe, C., Jouary, T., Schadendorf, D., Ribas, A., O'Day, S. J., Sosman, J. A., Kirkwood, J. 412 M., Eggermont, A. M., ... BRIM-3 Study Group (2011). Improved survival with 413 vemurafenib in melanoma with BRAF V600E mutation. The New England journal 414 of medicine, 364(26), 2507-2516.

415 https://doi.org/10.1056/NEJMoa1103782

417 18. Engelman, J. A., Zejnullahu, K., Mitsudomi, T., Song, Y., Hyland, C., Park, J. 418 O., Lindeman, N., Gale, C. M., Zhao, X., Christensen, J., Kosaka, T., Holmes, A. 419 J., Rogers, A. M., Cappuzzo, F., Mok, T., Lee, C., Johnson, B. E., Cantley, L. C., 420 \& Jänne, P. A. (2007). MET amplification leads to gefitinib resistance in lung 421 cancer by activating ERBB3 signaling. Science (New York, N.Y.), 316(5827), 422 1039-1043.

423 https://doi.org/10.1126/science.1141478

19. Jiapaer, S., Furuta, T., Tanaka, S., Kitabayashi, T., \& Nakada, M. (2018).

426 Potential Strategies Overcoming the Temozolomide Resistance for

427 Glioblastoma. Neurologia medico-chirurgica, 58(10), 405-421.

428 https://doi.org/10.2176/nmc.ra.2018-0141

430 20. Komarova N. (2006). Stochastic modeling of drug resistance in cancer. Journal 431 of theoretical biology, 239(3), 351-366. 
21. Gascoigne, K. E., \& Taylor, S. S. (2008). Cancer cells display profound intraand interline variation following prolonged exposure to antimitotic drugs. Cancer cell, 14(2), 111-122. https://doi.org/10.1016/j.ccr.2008.07.002 Review of Mathematical Models for Tumor Dynamics and Treatment Resistance Evolution of Solid Tumors. CPT: pharmacometrics \& systems

442 pharmacology, 8(10), 720-737.

443 https://doi.org/10.1002/psp4.12450

23. Foo, J., \& Michor, F. (2014). Evolution of acquired resistance to anti-cancer therapy. Journal of theoretical biology, 355, 10-20. https://doi.org/10.1016/j.jtbi.2014.02.025 \& Mukherjee, S. (2014). A stochastic model dissects cell states in biological transition processes. Scientific reports, 4, 3692. https://doi.org/10.1038/srep03692 cell plasticity. Nature, 501(7467), 328-337. cancer stem cell perspective. Molecular cancer, 16(1), 41. 
28. Tomasetti, C., \& Levy, D. (2010). An elementary approach to modeling drug resistance in cancer. Mathematical biosciences and engineering : MBE, 7(4), 905469918.

$470 \quad$ https://doi.org/10.3934/mbe.2010.7.905

472 29. Iwasa, Y., Nowak, M. A., \& Michor, F. (2006). Evolution of resistance during 473 clonal expansion. Genetics, 172(4), 2557-2566.

474 https://doi.org/10.1534/genetics.105.049791 Bovier, A. (2016). A stochastic model for immunotherapy of cancer. Scientific

478 reports, 6, 24169.

https://doi.org/10.1038/srep24169

32. Sun, X., Bao, J., \& Shao, Y. (2016). Mathematical Modeling of Therapy-

33. Figueredo, G. P., Siebers, P. O., Owen, M. R., Reps, J., \& Aickelin, U. (2014).

34. Avanzini, S., \& Antal, T. (2019). Cancer recurrence times from a branching process model. PLoS computational biology, 15(11), e1007423. 
503 36. Lee S. Y. (2016). Temozolomide resistance in glioblastoma multiforme. Genes

504 \& diseases, 3(3), 198-210.

505 https://doi.org/10.1016/j.gendis.2016.04.007

506

507 37. Perazzoli, G., Prados, J., Ortiz, R., Caba, O., Cabeza, L., Berdasco, M.,

508 Gónzalez, B., \& Melguizo, C. (2015). Temozolomide Resistance in Glioblastoma

509 Cell Lines: Implication of MGMT, MMR, P-Glycoprotein and CD133

510 Expression. PloS one, 10(10), e0140131.

511 https://doi.org/10.1371/journal.pone.0140131

513 38. Fan, C. H., Liu, W. L., Cao, H., Wen, C., Chen, L., \& Jiang, G. (2013). O6-

514 methylguanine DNA methyltransferase as a promising target for the treatment of

515 temozolomide-resistant gliomas. Cell death \& disease, 4(10), e876.

516 https://doi.org/10.1038/cddis.2013.388 


\section{Supplement}

\section{1. Effect of birth, death and transition rates in drug resistant cells}

531 Different cell birth, death and transition rates were examined to assess their effect

532 on population dynamics (Table $\mathrm{S} 1)$. A lower birth rate $\left(1.5^{*} \mathrm{n}\right)$ led to a smaller

533 resistant cell population in comparison to a higher birth rate $(2.5 * \mathrm{n})$ (Figure S1A-

534 B). To illustrate the effect of drug-induced time dependence on the death rate of

535 resistant cells, two different death rates $\left(\mathrm{e}^{-(\mathrm{t}-2) / 10}\right.$ and $\left.\mathrm{e}^{-(\mathrm{t}-2) / 100}\right)$ were used. In the first

536 case $\left(\mathrm{e}^{-(\mathrm{t}-2) / 10}\right)$, the death rate decreased rapidly leading to an exponential rise of

537 resistant cell population, whereas in the second case $\left(\mathrm{e}^{-(\mathrm{t}-2) / 100}\right)$, the death rate

538 decreased slowly, and thus resistant cells died at a faster rate (Figure S1C-D).

539 We next considered cell states under two conditions; either having no transitions or

540 having high transition rates $(2 * n)$. In the absence of transitions, there is no resistant

541 cell population as resistant cells arise from sensitive cells. Additionally, the cell state

542 trajectories remain independent of each other resulting in large disparity in the

543 number of cells occupying different cell states (Figure S1E). In the case of high

544 transition rates, resistant cells dominated the cell population. However, allowing

545 transitions between cells states changed the behavior of the system by allowing the

546 cells to move from more populated states to lesser-populated states (Figure S1F).

547 Hence, most of the cell state trajectories remained close to each other. 


\begin{tabular}{|c|c|c|c|c|c|c|c|c|c|c|c|}
\hline \multirow[t]{2}{*}{ Condition } & \multirow{2}{*}{$\begin{array}{l}\text { Time } \\
\text { (t) }\end{array}$} & \multicolumn{3}{|c|}{ Q Cells } & \multicolumn{3}{|c|}{$\begin{array}{c}\text { G-Tp, G-Tr } \\
\text { GS-Tp, GS-Tr }\end{array}$} & \multicolumn{3}{|c|}{ G-R, GS-R } & \multirow[t]{2}{*}{$\begin{array}{l}\text { Figure } \\
\text { Number }\end{array}$} \\
\hline & & $\mathbf{B R}$ & DR & TR & BR & DR & TR & BR & DR & TR & \\
\hline \multirow{2}{*}{$\begin{array}{l}\text { Low BR } \\
\text { of G-R, } \\
\text { GS-R }\end{array}$} & $t<2$ & 0 & $\mathrm{n}$ & $\mathrm{n}$ & $2 * n$ & $\mathrm{n}$ & $\mathrm{n}$ & NA & NA & NA & \multirow[b]{2}{*}{ Fig.S1A } \\
\hline & $t>2$ & 0 & $2.2 * \mathrm{n}$ & $\mathrm{n}$ & $2 * n$ & $2.2 * n$ & $\mathrm{n}$ & $1.5^{*} \mathrm{n}$ & $2.2 * \mathrm{n}^{*} \mathrm{e}^{-(\mathrm{t}-2) / 20}$ & $\mathrm{n}$ & \\
\hline \multirow{2}{*}{$\begin{array}{l}\text { High BR } \\
\text { of G-R, } \\
\text { GS-R }\end{array}$} & $t<2$ & 0 & $\mathrm{n}$ & $\mathrm{n}$ & $2 * n$ & $\mathrm{n}$ & $\mathrm{n}$ & NA & NA & NA & \multirow[b]{2}{*}{ Fig.S1B } \\
\hline & $t>2$ & 0 & $2.2 * \mathrm{n}$ & $\mathrm{n}$ & $2 * n$ & $2.2 * n$ & $\mathrm{n}$ & $2.5^{*} \mathrm{n}$ & $2.2 * \mathrm{n}^{*} \mathrm{e}^{-(\mathrm{t}-2) / 20}$ & $\mathrm{n}$ & \\
\hline \multirow{2}{*}{$\begin{array}{l}\text { Low DR } \\
\text { of G-R, } \\
\text { GS-R }\end{array}$} & $t<2$ & 0 & $\mathrm{n}$ & $\mathrm{n}$ & $2 * n$ & $\mathrm{n}$ & $\mathrm{n}$ & NA & NA & NA & \multirow[b]{2}{*}{ Fig.S1C } \\
\hline & $t>2$ & 0 & $2.2 * \mathrm{n}$ & $\mathrm{n}$ & $2 * n$ & $2.2 * \mathrm{n}$ & $\mathrm{n}$ & $2 * n$ & $2.2 * \mathrm{n}^{*} \mathrm{e}^{-(\mathrm{t}-2) / 10}$ & $\mathrm{n}$ & \\
\hline \multirow{2}{*}{$\begin{array}{l}\text { High DR } \\
\text { of G-R, } \\
\text { GS-R }\end{array}$} & $\mathrm{t}<2$ & 0 & $\mathrm{n}$ & $\mathrm{n}$ & $2 * n$ & $\mathrm{n}$ & $\mathrm{n}$ & NA & NA & NA & \multirow[b]{2}{*}{ Fig.S1D } \\
\hline & $t>2$ & 0 & $2.2 * \mathrm{n}$ & $\mathrm{n}$ & $2 * n$ & $2.2 * \mathrm{n}$ & $\mathrm{n}$ & $2 * n$ & $2.2 * \mathrm{n} * \mathrm{e}^{-(\mathrm{t}-2) / 100}$ & $\mathrm{n}$ & \\
\hline \multirow{2}{*}{$\begin{array}{l}\text { No } \\
\text { Transition } \\
\text { rate }\end{array}$} & $\mathrm{t}<2$ & 0 & $\mathrm{n}$ & 0 & $2 * n$ & $\mathrm{n}$ & 0 & NA & NA & NA & \multirow[b]{2}{*}{ Fig.S1E } \\
\hline & $t>2$ & 0 & $2.2 * \mathrm{n}$ & 0 & $2 * n$ & $2.2 * \mathrm{n}$ & 0 & $2 * n$ & $2.2 * \mathrm{n} * \mathrm{e}^{-(\mathrm{t}-2) / 20}$ & 0 & \\
\hline \multirow{2}{*}{$\begin{array}{l}\text { High } \\
\text { Transi- } \\
\text {-tion rate }\end{array}$} & $t<2$ & 0 & $\mathrm{n}$ & $2 * n$ & $2^{*} \mathrm{n}$ & $\mathrm{n}$ & $2 * n$ & $\mathrm{NA}$ & NA & NA & \multirow[b]{2}{*}{ Fig.S1F } \\
\hline & $t>2$ & 0 & $2.2 * \mathrm{n}$ & $2 * n$ & $2 * n$ & $2.2 * \mathrm{n}$ & $2 * n$ & $2 * n$ & $2.2 * \mathrm{n} * \mathrm{e}^{-(\mathrm{t}-2) / 20}$ & $2 * n$ & \\
\hline
\end{tabular}

549 Table S1: Summary of model simulation conditions with alternate birth, death, and

550 transition rates. $n$ indicates the number of cells in a state; $t$ is the time elapsed in the

551 system. BR: Birth rate, DR: Death rate and TR: transition rate. 


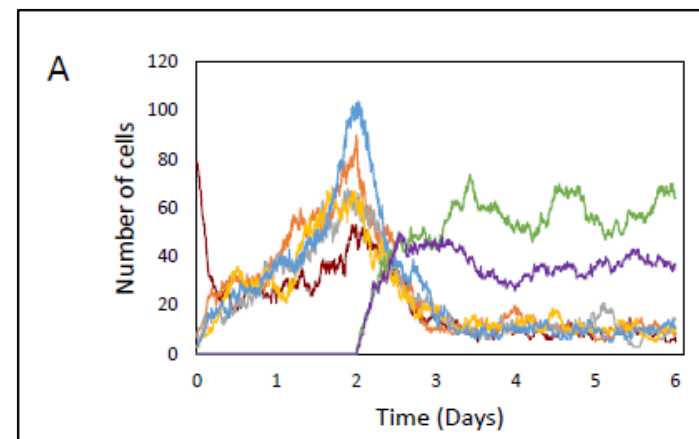

B

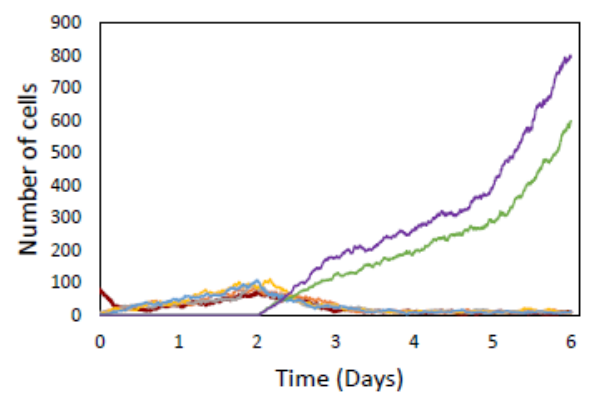

C

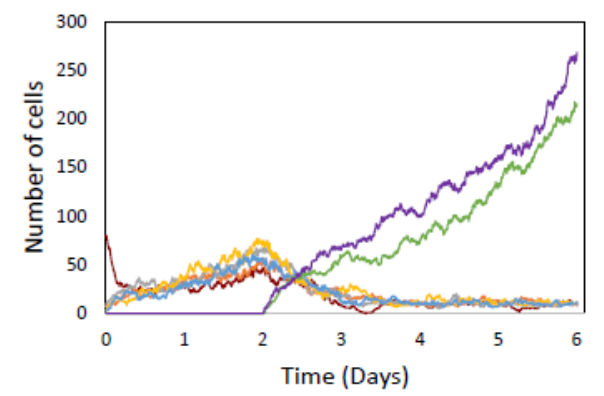

D

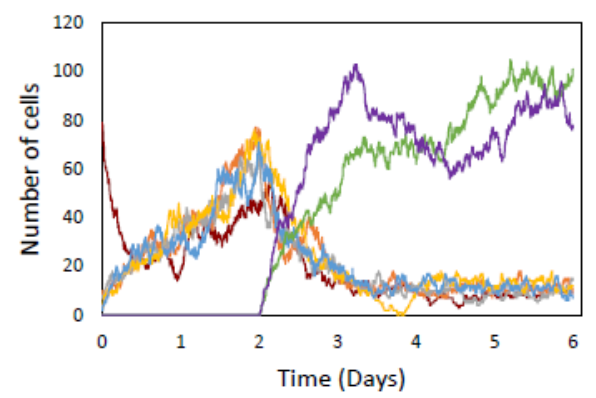

$E$

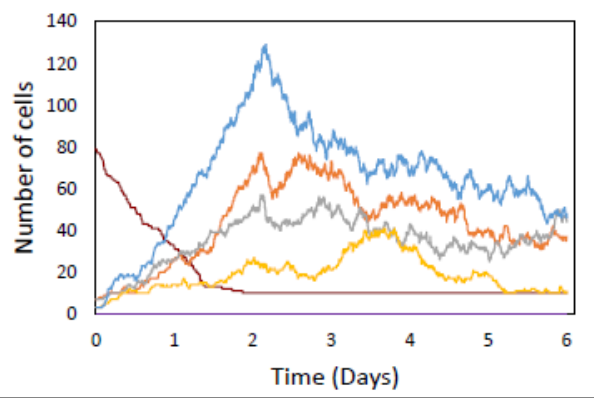

$\mathrm{F}$

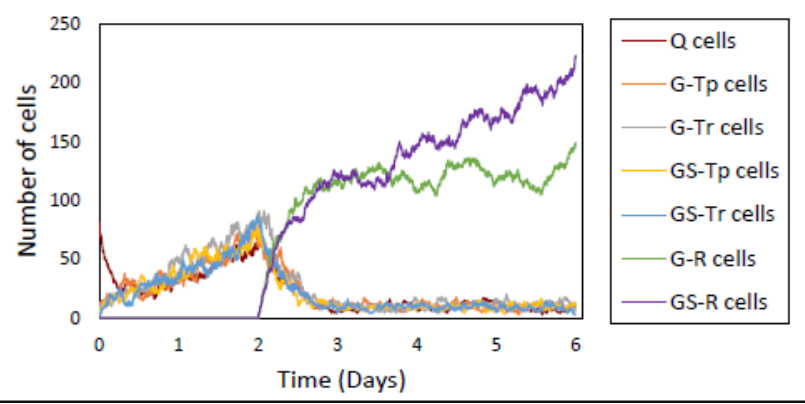

554 Figure S1: Effects of changing birth/death/transition rates on cell population

555 dynamics. (A) Simulation for low birth rate of resistant cells $\left(1.5^{*} n\right)$. (B) Simulation

556 for high birth rate of resistant cells $\left(2.5^{*} \mathrm{n}\right)$. (C) Simulation for low death rate of

557 resistant cells $\left(2.2 * \mathrm{n}^{*} \mathrm{e}^{-(\mathrm{t}-2) / 10}\right)$. (D) Simulation for high death rate of resistant cells

$558\left(2.2 * n^{*} \mathrm{e}^{-(\mathrm{t}-2) / 100}\right)$. (E) Simulation without any transition. (F) Simulation with high

559 transition rate $(2 * n)$. 
As stated before, in our model, there are seven cell states, which are denoted as a row vector $\left\{n_{1}, n_{2}, \ldots, n_{7}\right\} \quad\left(n_{i}\right.$ represent the number of cells in the $i^{\text {th }}$ state). We assume continuous time Markovian dynamics for cell proliferation, which means that the future evolution of the system can be predicted from the current state alone

566 (memoryless). The process of birth, death, and transition between any cell states $i$

567 and $j$ can be written as

568 Birth: $n_{i} \stackrel{c_{b i}}{\longrightarrow} n_{i}+1 \quad$ Death: $n_{i} \stackrel{c_{d i}}{\longrightarrow} n_{i}-1$

569 Transitions: $n_{i} \stackrel{c_{t_{i j}}}{\longrightarrow} n_{i}-1 \quad n_{j} \stackrel{c_{t_{i j}}}{\longrightarrow} n_{j}+1$

571 where $c_{b i}$ and $c_{d i}$ is the birth and death rate coefficient in $i^{\text {th }}$ cell state, respectively.

$572 c_{t_{i j}}$ is the transition rate coefficient regarding the transition from $i^{t h} \rightarrow j^{\text {th }}$ cell state.

573 All of the above processes can be written in terms of transitional probabilities (Figure

$574 \quad$ S2) as

575 Birth:

$$
\begin{aligned}
& P\left(n_{1}+1, n_{2}, \ldots, n_{7} ; t+\Delta t \mid n_{1}, n_{2}, \ldots, n_{7} ; t\right)=c_{b 1} n_{1} \Delta t \\
& P\left(n_{1}, n_{2}+1, \ldots, n_{7} ; t+\Delta t \mid n_{1}, n_{2}, \ldots, n_{7} ; t\right)=c_{b 2} n_{2} \Delta t
\end{aligned}
$$

577

578

$$
P\left(n_{1}, n_{2}, \ldots, n_{7}+1 ; t+\Delta t \mid n_{1}, n_{2}, \ldots, n_{7} ; t\right)=c_{b 7} n_{7} \Delta t
$$


Death:

$$
P\left(n_{1}-1, n_{2}, \ldots, n_{7} ; t+\Delta t \mid n_{1}, n_{2}, \ldots, n_{7} ; t\right)=c_{d 1} n_{1} \Delta t
$$

$$
P\left(n_{1}, n_{2}-1, \ldots, n_{7} ; t+\Delta t \mid n_{1}, n_{2}, \ldots, n_{7} ; t\right)=c_{d 2} n_{2} \Delta t
$$

$$
P\left(n_{1}, n_{2}, \ldots, n_{7}-1 ; t+\Delta t \mid n_{1}, n_{2}, \ldots, n_{7} ; t\right)=c_{d 7} n_{7} \Delta t
$$

$$
P\left(n_{1}-1, n_{2}+1, \ldots, n_{7} ; t+\Delta t \mid n_{1}, n_{2}, \ldots, n_{7} ; t\right)=c_{t_{12}} n_{1} \Delta t
$$

$$
P\left(n_{1}+1, n_{2}-1, \ldots, n_{7} ; t+\Delta t \mid n_{1}, n_{2}, \ldots, n_{7} ; t\right)=c_{t_{21}} n_{2} \Delta t
$$

$$
P\left(n_{1}, \ldots, n_{6}-1, n_{7}+1 ; t+\Delta t \mid n_{1}, n_{2}, \ldots, n_{7} ; t\right)=c_{t_{67}} n_{6} \Delta t
$$

$$
P\left(n_{1}, \ldots, n_{6}+1, n_{7}-1 ; t+\Delta t \mid n_{1}, n_{2}, \ldots, n_{7} ; t\right)=c_{t_{76}} n_{7} \Delta t
$$


600 transitions defined above, we can write the probability distribution of the state

$601\left\{n_{1}, \ldots, n_{6}, n_{7}\right\}$ at time $t+\Delta t$ as

602

603

$P\left(n_{1}, \ldots, n_{6}, n_{7} ; t+\Delta t\right)=c_{b 1}\left(n_{1}-1\right) \Delta t P\left(n_{1}-1, \ldots, n_{6}, n_{7} ; t\right)+c_{b 2}\left(n_{2}-\right.$

604

1) $\Delta t P\left(n_{1}, n_{2}-1, \ldots, n_{7} ; t\right)+\cdots+c_{d 1}\left(n_{1}+1\right) \Delta t P\left(n_{1}+1, \ldots, n_{6}, n_{7} ; t\right)+$

605

$c_{d 2}\left(n_{2}+1\right) \Delta t P\left(n_{1}, n_{2}+1, \ldots, n_{7} ; t\right)+\cdots+c_{t_{12}}\left(n_{1}+1\right) \Delta t P\left(n_{1}+1, n_{2}-\right.$

606

$\left.1, \ldots, n_{7} ; t\right)+c_{t_{21}}\left(n_{2}+1\right) \Delta t P\left(n_{1}-1, n_{2}+1, \ldots, n_{7} ; t\right)+c_{t_{13}}\left(n_{1}+\right.$

607

1) $\Delta t P\left(n_{1}+1, n_{2}, n_{3}-1, \ldots, n_{7} ; t\right)+c_{t_{31}}\left(n_{3}+1\right) \Delta t P\left(n_{1}-1, n_{2}, n_{3}+\right.$

608

$\left.1, \ldots, n_{7} ; t\right)+\cdots+(1-\alpha) \Delta t P\left(n_{1}, \ldots, n_{6}, n_{7} ; t\right)$

609

610 where $\alpha$ is the sum of all transition probabilities from other states to the state $\left\{n_{1}, \ldots, n_{6}, n_{7}\right\}$. The above equation can be written in a succinct form as

612

613

$$
P\left(n_{1}, \ldots, n_{6}, n_{7} ; t+\Delta t\right)
$$

614

$$
=\sum_{i} c_{b i}\left(n_{i}-1\right) \Delta t P\left(n_{i}-1 ; t\right)+\sum_{i} c_{d i}\left(n_{i}+1\right) \Delta t P\left(n_{i}+1 ; t\right)
$$

615

$$
+\sum_{i, j} c_{t_{i j}}\left(n_{i}+1\right) \Delta t P\left(n_{i}+1, n_{j}-1 ; t\right)
$$

616

$$
+(1-\alpha) \Delta t P\left(n_{1}, \ldots, n_{6}, n_{7} ; t\right)
$$


618 Applying a few algebraic manipulations yields the master equation as

619

$620 \frac{d P\left(n_{i}\right)}{d t}=\sum_{i} c_{b i}\left(n_{i}-1\right) P\left(n_{i}-1 ; t\right)+\sum_{i} c_{d i}\left(n_{i}+1\right) P\left(n_{i}+1 ; t\right)+$

621

$$
\sum_{i, j} c_{t_{i j}}\left(n_{i}+1\right) P\left(n_{i}+1, n_{j}-1 ; t\right)-\alpha P\left(n_{1}, \ldots, n_{6}, n_{7} ; t\right)
$$

622

623 The first term corresponds to the birth, the second term to death, the third term

624 represents outward and inward transitions from and to a particular state, and the last

625 term is the probability of none of the above events occurring. Both birth and death

626 rates are proportional to the number of cells in the corresponding state. The transition

627 rate is proportional to the number of cells in the initial state $\left(n_{i}\right)$. The coefficients

628 are set according to the birth rates, death rates, and transition rates between the states.

629 Once the initial values of $\left(n_{i}\right)$ and coefficients $\left(c_{b i}, c_{d i}, c_{t_{i j}}\right)$ are known, the

630 stochastic time evolution of the system can be evaluated using the master equation. 
633 Figure S2: Illustration of the flow of cell state probability within the cell state

634 space through the process of birth, death, and transition. $n_{i}$ Indicates the number of

635 cells in the $i^{\text {th }}$ state. $c_{b i}, c_{d i}$ and $c_{t_{i j}}$ are birth, death and transition rate coefficient, 636 respectively.

\section{Simulations}

639 To simulate the system, the Gillespie algorithm was used, where individual 640 trajectories are simulated instead of the whole probability distribution. The 641 algorithm assumes the processes to be Markovian and a time step $(\tau)$ is used based 642 on the probability of the event occurrence (Poisson distribution). To find $\tau$, we 643 define the propensity function as

$$
a_{0}=\sum_{i} a_{i}
$$


645 where the probability that any given event will occur per unit time is $a_{i}$. The time

646 before the next reaction occurs is a random variable with distribution

647

648

$$
p(\tau)=a_{0} e^{-a_{0} \tau}
$$

650 We can generate an exponentially distributed $\tau$ (time to next reaction) by

$$
\tau=\frac{1}{a_{0}} \ln \left(\frac{1}{r_{1}}\right)
$$

653 where, $r_{1}$ is a uniformly distributed random number in $(0,1)$. We used another 654 random number to select which of the reactions (based on their weight) is going to

655 take place. If event 1 is selected: $n_{1} \rightarrow n_{1}+1$. If event 2 is selected: $n_{1} \rightarrow n_{1}-1$

656 and so on. We update the number of cells in each cell state and repeat the process

657 until the desired time or maximum cell population is reached. The code is available 658 on GitHub (https://github.com/anshulsa/Cell_state_transition.git). 